September 2019 to January 2020. Adults with EVH were included in the study. The clinical characteristics and laboratory data at admission were documented, based on which MELD and CTP scores were calculated. The surviving patients were then followed via telephone after 30 days and readmission and its reasons, mortality, and morbidity within 30-days were determined.

Results A total of $95 \mathrm{EVH}$ patients were included in the study, out of which $74.7 \%$ were males. The mean age of the participants was 49.56 years. The etiology was Hepatitis C in $62(65.3 \%)$ patients. The in-hospital mortality was $5(5.3 \%)$. Of those who survived, $17(17.5 \%)$ had readmissions with rebleeding as cause in $7(7.4 \%)$ patients. The rest of the patients were admitted with other complications of end-stage liver disease.

Conclusions The all-cause 30-day readmission rate after EVH was $17.5 \%$ with more than one-third of the cases due to rebleeding. The readmission was not associated with higher rates of mortality (in-hospital mortality rate vs readmission mortality rate).

\section{IDDF2020-ABS-0176 CLINICIAN EXPERIENCE AND ATTITUDES TO PALLIATIVE CARE IN PATIENTS WITH HCC - AN AUSTRALIA-WIDE SURVEY}

${ }^{1}$ Abdul-Hamid Sabih*, '2Lynn Lim, ${ }^{2}$ Maria Cigolini, ' Simone I Strasser, ${ }^{1}$ Ken Liu. ${ }^{1} A W$ Morrow Gastroenterology and Liver Centre, Royal Prince Alfred Hospital, Australia; ${ }^{2}$ Palliative Care Services, Royal Prince Alfred Hospital, Australia

\subsection{6/gutjpl-2020-IDDF.162}

Background Palliative care (PC) service involvement in HCC patients is suboptimal. Little is known about clinician experience and attitudes towards PC in HCC, which formed the aim of our study.

Methods A nationwide survey of consultants/trainees was conducted through the Gastroenterological Society of Australia. Clinician and practice demographics, experience and attitudes towards PC use in HCC patients were collected.

Results 161 respondents participated with representation from all states/territories (61\% male, 94\% gastroenterologist/hepatologist). Most worked in public metropolitan hospitals (79\%) with weekly multidisciplinary tumour board meetings (MDTBM) (59\%) and had no formal PC training (71\%).
MDTBM with PC team attendance was reported by $11 \%$, although $77 \%$ thought this would be useful. Both rates of PC referral and perceived usefulness of PC increased incrementally from Barcelona Clinic Liver Cancer (BCLC) 0/A to BCLC D patients but were not universal even in advanced $(46 \%) /$ terminal $(87 \%)$ stages. Those with prior PC training were more likely to refer BCLC 0/A patients for early PC $(P=0.01)$. Referral rates for outpatient $\mathrm{PC}$ were higher in respondents who attended MDTBM with PC present $(P<0.05$ for all BCLC stages). Common reasons for referral were: end-of-life care (93\%), pain (63\%), treatment sideeffects (21\%) and psychological symptoms (21\%). Most acknowledged PC discussions with patients occurred too late (61\%) while the best time was thought to be at diagnosis of an incurable disease (61\%). PC service was rated good/very good by $70 \%$ for outpatients and $81 \%$ for inpatients and $81 \%$ thought the referral process was easy. Major barriers identified to PC referral were: negative associations with the term 'PC' (83\%), patient/family lack of acceptance $(82 \%$ / $77 \%)$, cultural factors (74\%) and insufficient time in clinic (70\%). The majority (78\%) thought patients would be more accepting of PC if the name was changed to 'supportive care'.

Conclusions PC referral for HCC patients occurs late and is not universal even in late-stage disease. Barriers to PC referral were not related to the quality of/access to PC services but rather to clinician perception/belief that PC would not be accepted by patients and their families.

\section{IDDF2020-ABS-0180 MULTIPLE NODULAR LIVER MASSES IN ELDERLY PATIENT WITH NON-CIRRHOTIC HEPATITIS C: A DILEMMA BETWEEN HEPATOCELLULAR CARCINOMA AND LIVER ABSCESS}

Jeremia Immanuel Siregar*, Ignatius Bima Prasetya, Nata Pratama Hardjo Lugito, Andree Kurniawan. Department of Internal Medicine, Faculty of Medicine, Pelita Harapan University, Indonesia

\subsection{6/gutjnl-2020-IDDF.163}

Background Unusual appearance of liver masses poses diagnostic challenges in differentiating between malignancy and abscess. Here, we found an indeterminate case of liver masses
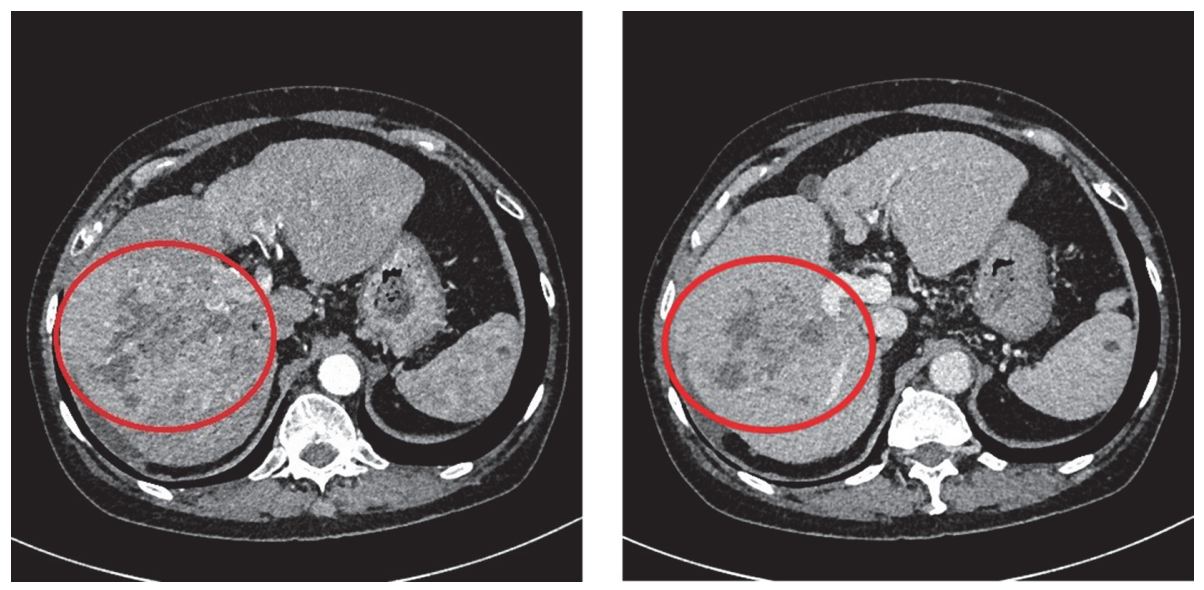

Abstract IDDF2020-ABS-0180 Figure 1 Arterial and venous phase from abdominal CT of the patient, showing large liver mass with perihepatic fluids and multiple cystic lesions in various sizes that were conglomerated inside the mass 
from an elderly patient with non-cirrhotic hepatitis $\mathrm{C}$ that needs further evaluations.

Methods A 78-year-old man was admitted due to right upper quadrant pain, mild fever and weight loss. Triple-phase abdominal CT showed a large liver mass $(12.8 \times 8.6 \times 10.2$ $\mathrm{cm}$ in size) with perihepatic fluids and multiple cystic lesions in various sizes that were conglomerated inside the mass (figure 1). Multiple satellites nodules with central cysts were also found, suggesting either hepatocellular carcinoma (HCC) with intrahepatic metastasis or multiple liver abscesses. He had elevated leucocytes $\left(15,570 \mathrm{~mm}^{-3}\right)$ and alanine transaminase $(244$ $\mathrm{U} / \mathrm{L}$ ). However, his serum seramoeba and fecal amoeba tests were negative. His Anti-HCV serology was positive. Moreover, his AFP and CEA levels were $75.94 \mathrm{ng} / \mathrm{mL}$ and $1.8 \mathrm{ng} / \mathrm{mL}$ consecutively. Two weeks course of antibiotics were initiated with no changes in his liver masses after further evaluation of abdominal CT. However, his leucocytes and alanine transaminase improved towards normal levels. Liver biopsy was offered for the patient, but he refused to do so.

Results The presentation of HCC can sometimes mimic an abscess, especially when cystic degeneration appeared within the tumor. Meanwhile, there were some cases of liver abscess that were indistinguishable from HCC. The patient, in this case, had fever and leucocytosis, which were the characteristics for liver abscess. Moreover, his AFP level was not increased significantly. However, insignificant changes after antibiotic therapy raised a dilemma on his definite diagnosis. Although very rare, a non-cirrhotic hepatocellular carcinoma in hepatitis $\mathrm{C}$ can also occur. This direct carcinogenesis mechanism can be potentially induced by the non-structural protein of hepatitis $\mathrm{C}$ virus. Looking at this case, a liver biopsy is needed for concluding a diagnosis for this patient.

Conclusions Establishing a diagnosis for atypical presentations of HCC and liver abscess can be quite difficult. Due to the big differences in their prognosis, familiarity with unusual clinical and imaging findings, as well as liver biopsy, are needed to ensure a certain diagnosis for the patient.

\section{IDDF2020-ABS-0189 CLINICAL ANALYSIS OF LAPS IN THE TREATMENT OF HEPATITIS B VIRUS- RELATED HEPATOCELLULAR CARCINOMA}

Zebin Chen*, Kai Lei, Shunli Shen, Baogang Peng. Department of Liver Surgery, the First Affiliated Hospital, Sun Yat-sen University, China

\subsection{6/gutjnl-2020-IDDF.164}

Background The aim of the study is to assess the efficacy and safety of laparoscopic microwave ablation and portal vein ligation for staged hepatectomy (LAPS) in the treatment of hepatitis B virus-related hepatocellular carcinoma.

Methods Clinical data of patients with hepatitis B virus-related liver cancer who underwent LAPS or ALPPS surgery in the First Affiliated Hospital of Sun Yat-sen University from April 2013 to September 2019 were retrospectively analyzed. In the study, all patients signed informed consents and were approved by local ethics committees. Statistical analysis of data were performed by the SPSS 26.0.

Results 7 patients with HCC underwent LAPS, while 14 patients underwent ALPPS surgery during the same period. $42.9 \%$ of patients had a single tumor, and the median tumor diameter was $96 \mathrm{~mm}$ (range: $26-151 \mathrm{~mm}$ ), the median preoperative FLR was 29.0\% (range19.5\%-38.8\%). The severe morbidity rates of patients undergoing LAPS was lower than those receiving ALPPS after the first operation $(P=0.009)$. The median time to first passage of flatus of patients in the ALPPS group and LAPS group was $3 \mathrm{~d}$ and $2 \mathrm{~d}(P=0.037)$ and the median time to out of bed was $4 \mathrm{~d}$ and $2 \mathrm{~d}(P=0.040)$. 6 patients in the LAPS group completed the second stage operation, and 11 patients in the ALPPS group completed the second stage operation $(P=0.694)$. The median interval was 15 days in the LAPS group and 11 days in the ALPPS group $(P=0.006)$. The incidence of severe complications was $36.4 \%$ in the ALPSS group and $50.0 \%$ in the LAPS group after the second operation $(P=0.615)$. One patient in the ALPPS group died after the second operation. There was no difference in FLR growth rate, operative time and intraoperative loss between the two groups.

Conclusions LAPS has potential advantages over classical ALPPS in reducing postoperative liver failure and severe complications. LAPS may have the same effect in promoting the growth of FLR in patients with hepatitis B virus-related HCC, with a longer interval between the two operations.

\section{IDDF2020-ABS-0192 TERLIPRESSIN NON-RESPONSE PREDICTS MORTALITY IN ACUTE-ON-CHRONIC LIVER FAILURE-A PROSPECTIVE COHORT STUDY}

Anand Kulkarni*, TR Sowmya, Mithun Sharma, Pramod Kumar, Samragni Vasireddy, Harshavardhan Tevethia, Nagaraja Rao Padaki, D Nageshwar Reddy. AlG Hospitals, Asian Institute of Gastroenterology, India

\subsection{6/gutjnl-2020-IDDF.165}

Background Terlipressin with albumin is the recommended standard of care for hepatorenal syndrome. However, there is a paucity of Asian data on the use of terlipressin in acute-onchronic liver failure (ACLF) patients. We aimed to evaluate the safety and efficacy of terlipressin infusion in ACLF patients with HRS-AKI (acute kidney injury).

Methods Consecutive ACLF patients aged between 18-75 years treated with terlipressin infusion for HRS-AKI were included. The primary objective was to assess the incidence of adverse events, and secondary were to assess the response to terlipressin therapy, predictors of terlipressin non-response, and transplant-free survival at day 30, 90.

Results During the study period, 116 ACLF patients (Males-

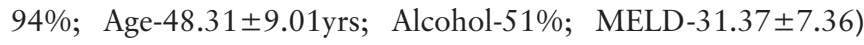
received terlipressin therapy for HRS-AKI. Fifty-two percent had a bacterial infection at baseline. The mean dose of terlipressin was $2.75 \pm 0.93 \mathrm{mg} /$ day for $5.28 \pm 3.51$ days. Twentyone percent of patients developed adverse effects (Diarrhea$37.5 \%$, abdominal pain-25\%) to terlipressin, and $12 \%$ required drug discontinuation. Sixty-five percent $(75 / 116)$ of patients responded to terlipressin, of which $39.7 \%(46 / 116)$ had a complete response, and 25\% (29/116) achieved a partial response. Time to the reversal of AKI was $4.8 \pm 2.64$ days. Twenty-eight percent of patients required renal replacement therapy. Transplant free survival at day 30 and 90 was $71 \%$ and $57.8 \%$. On multivariate analysis baseline serum creatinine [OR-2.24 (1.41-3.57);p<0.001], ACLF grade [Gr.II-4.98 (1.5$16.5) ; \mathrm{p}=0.009$, Gr.III-7.61 (1.91-30.16); $\mathrm{p}=0.004]$, and change in MAP at day 3 [OR-0.73 $(0.57-0.92) ; \mathrm{p}=0.009]$ were predictors of terlipressin non-response. On multivariate analysis terlipressin non-response [HR-3.49 (1.85-6.57); p<0.001] and 